\title{
Virtual Reality Surgery Simulation: A Survey on Patient Specific Solution
}

\author{
Jinglu Zhang, Jian Chang, Xiaosong Yang, and Jianjun Zhang* \\ National Center for Computer Animation, Bournemouth University, Poole, UK \\ \{zhangj, jchang, xyang, jzhang\}@bournemouth.ac.uk
}

\begin{abstract}
For surgeons, the precise anatomy structure and its dynamics are important in the surgery interaction, which is critical for generating the immersive experience in VR based surgical training applications. Presently, a normal therapeutic scheme might not be able to be straightforwardly applied to a specific patient, because the diagnostic results are based on averages, which result in a rough solution. Patient Specific Modeling (PSM), using patient-specific medical image data (e.g. CT, MRI, or Ultrasound), could deliver a computational anatomical model. It provides the potential for surgeons to practice the operation procedures for a particular patient, which will improve the accuracy of diagnosis and treatment, thus enhance the prophetic ability of VR simulation framework and raise the patient care. This paper presents a general review based on existing literature of patient specific surgical simulation on data acquisition, medical image segmentation, computational mesh generation, and soft tissue real time simulation.
\end{abstract}

Keywords: Patient Specific Modeling, Surgery Simulation, Virtual Reality.

\section{Introduction}

Approximately 200,000-injury or death cases come from preventable human medical errors in hospitals annually [15].Undesirable effects caused by human technique errors during surgeries and the fast development of new approaches (such as minimally invasive surgery), which requires more complex technical proficiency, have significantly emphasized the importance of surgical skills training in a secure and reusable environment. With the rapid explosion of Virtual Reality (VR) technology, the VR based surgery simulator provides the solution of teaching and assessing training skills outside the operation room with high efficiency and low risks [11]. Examples of commercial high-fidelity VR simulators, including Neuro Touch, LapSim [16], and Lap Mentor [58], are inclusive training systems consisting of laparoscopic and endoscopic surgery, general surgery, and bariatric surgery.

Usually, a treatment might not be able to directly applied to a specific patient due to the fact that the diagnostic results are based on averages, which

\footnotetext{
* Corresponding author
} 
result in a rough solution $[32,52]$. PSM could handle medical image data (e.g. CT, MRI, or Ultrasound), geometry, and material properties by anatomically accurate means. It presents the potential for surgeons to practice the surgical procedure preoperatively using VR simulator with the precise anatomy structure and its dynamics for an individual patient [46]. This concept would improve the accuracy of diagnosis and treatment, enhance the predictability of VR based surgery simulation framework, and raise the patient care $[4,40,44]$. In recent years, several surgical systems in laparoscopic nephrectomy [38], wrist joint [18, 19], hepatectomy and pancreatectomy [17] have combined the patient specific characteristics into the simulator.

However, it is still challenging for the state of the art algorithms in computer graphics and computer vision fields to bring patient specific modeling into standard clinical usage concerning too many manual steps involved from data acquisition, segmentation, 3D mesh generation to final surgery simulation $[22,28$, 48]. The nonlinearity and complexity of their biomechanical models also cause extreme challenges to give haptic feedbacks in real-time. Automating existing manual steps in the workflow (such as soft-tissue segmentation, labelling, and geometric corrections), identifying the complicated patient-specific material properties, and creating a reusable and extendable pre-modeled prototype database are indispensable for the future clinical approval. A general patient-specific workflow is shown in Fig.1. This survey focuses on comparing and discussing current progress in medical image segmentation, mesh generation, and soft tissue simulation.

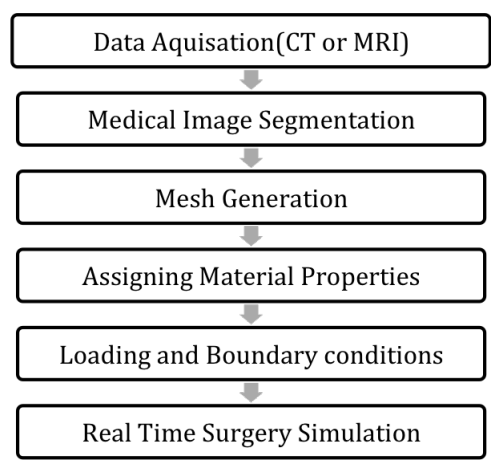

Fig. 1. General workflow of patient-specific modelling

\section{Geometry and Material Properties Acquisition}

Patient-specific modeling takes the advantage of personal geometry and material properties to generate the accurate computational mesh. There are primarily two techniques to obtain the necessary parameters for a tissue or an organ. The 
first way is directly extracting the information from 3D medical images, namely Computed tomography (CT), Magnetic Resonance (MR), three-dimensional ultrasound (3D-US), positron emission tomography (PET) and rotational angiography (RA) scanners. These kinds of medical images could provide anatomical information represented as a 3D array of grey scale intensities in Digital Imaging and Communications in Medicine (DICOM) format [3]. Meshes are first generated from the image preprocessing and then assigned the material properties to create the computational model.

3D image segmentation could also be conducted in a top-down fashion by forming, training, and matching statistic models with shape and appearance variations. The approach of concentrating on landmark-based shape representation and variants of Active Shape [14] and Active Appearance [13] models are known as Statistic shape models (SSM) [26]. Comparing with the first method, SSM could prevent the risks correlated with CT scans [6], largely save the computational cost for the sake of fewer image preprocessing steps involved, and the final output model could be put into the pre-model database for the future application. However, studies in SSM field are still immature and need further validation, so this review mainly discusses the patient-specific modeling through the medical image processing.

Although there are different modalities of medical images, this review focuses on CT and MRI based methods considering they are the most widely accepted non-invasive radiographic techniques. Comparing with MRI, CT is well suited for bone injuries, cancer detection, and lung and chest diagnose. Whereas MRI could produce clearer differences between normal and abnormal tissues, it is more suitable for soft tissue evolution studies such as brain tumors and spinal cord injury. Table 1 compares the difference of characteristics between CT and MRI.

Table 1. Comparison between CT and MRI

\begin{tabular}{|c|l|l|}
\hline Image Types & \multicolumn{1}{|c|}{ Characteristics } & \multicolumn{1}{|c|}{ Appropriate Study Areas } \\
\hline \multirow{3}{*}{ CT } & 1. Time and cost efficient & 1. Bone injuries \\
& 2. Painless & 2. Cancer detection \\
& 3. Shows up the acute bleed & 3. Lung and chest diagnose \\
\hline \multirow{3}{*}{ MRI } & 1. No radiation exposure & 1. Brian tumors \\
& 2. Good resolution & 2. Ligament and tendon injuries \\
& 3. Clearer differences between & 3. Spinal cord injury \\
& normal and abnormal tissues & \\
\hline
\end{tabular}

\section{Segmentation}

Segmentation, a process of dividing medical images into regions with similar properties such as color, texture, contrast, and gray level that specifically dis- 
play different tissue structures, organs, or pathologies, is critical for 3D anatomical reconstruction in PSM pipeline [23]. Segmentation has been regarded as the most tedious step for the reason that human anatomical structures are naturally complicated and rarely own any linear features. Even the segmentation of bone, the tissue that is considered as physically and geometrically linear, faces obstacles like the similar intensity with adjacent soft tissues and different grey values for different bone regions [33]. While various algorithms have been proposed in recent years, to achieve complete, accurate, and efficient automatic segmentation is still a tough problem. Medical image segmentation algorithms could be roughly divided into three categories: edge based segmentation, region based segmentation, and clustering and classification based segmentation.

\subsection{Edge based Segmentation}

Edge based segmentation generally applies the derivative operator to search and identify gradient fields in images. This conventional method separates the objects boundaries by locating sharp discontinuities in different color or gray level information. Prewitt [51] provides the first order derivative edge-detecting operator to approximate the magnitude and orientation of an image limited in the $3 \times 3$ district for eight directions. Second order derivative edge detective introduced by Marr and Hildreth in 1980 [39] who suggest applying Gaussian smoothing before Laplacian. Edge based segmentation methods are noise-sensitive. Sometimes the fake edge and weak edge could not be detected so that they always need to be combined with the region based segmentation algorithms.

\subsection{Region based Segmentation}

Compared with the edge-based segmentation that segments images depending on sharp changes between edges, region-based segmentation partitions images into homogeneous regions according to predefined rules. They are rather simple and noise immune.

Region Growing. Starting with a seed region and the stop condition (gray level intensity, shape, color, texture or model), regions growing by appending pixels that share similar properties to the seed region, and finally, stop when no more pixel meets the predefined growing criteria [1].

Region Splitting and Merging. Region splitting and merging [29] the conjunction of splitting and merging algorithms. The former algorithm sets an initial region includes the whole image, then iteratively splits regions into sub-regions referring to similarity criteria, and stops when no more splitting is possible. Merging algorithm complies with a contrast rule with splitting techniques by repeatedly merging similar region with similarity criteria. Region-based approaches hugely rely on seed regions according to the selection and the similarity principles, consequently, it might have the chance to over or under segment and waste computational costs both on time and memory. 


\subsection{Clustering and Classification}

Supervised learning. In 1993, Pal and Pal [49] predicted that Artificial Neural Network (ANN) based algorithms would be widely used in image segmentation. ANN, a self-learning model consists of large number of connected simple units called Artificial Neurons, could offer real-time automatic medical image segmentation by training classifier using training set (extracting features) in advance. Latterly, various supervised, semi-supervised, and unsupervised learning algorithms are developed. Recently, Havaei et al. [25] propose an automatic brain tumor segmentation method based on deep neural networks which achieve very fast segmentation between 25 seconds to 3 minutes, the sample result is shown in Figure. 2 The biggest problem for all machine learning methods is that the learning methods are not able to formulate the model far outside the data collected during the training stage, so this made the data capture the crucial part of the supervised methods.

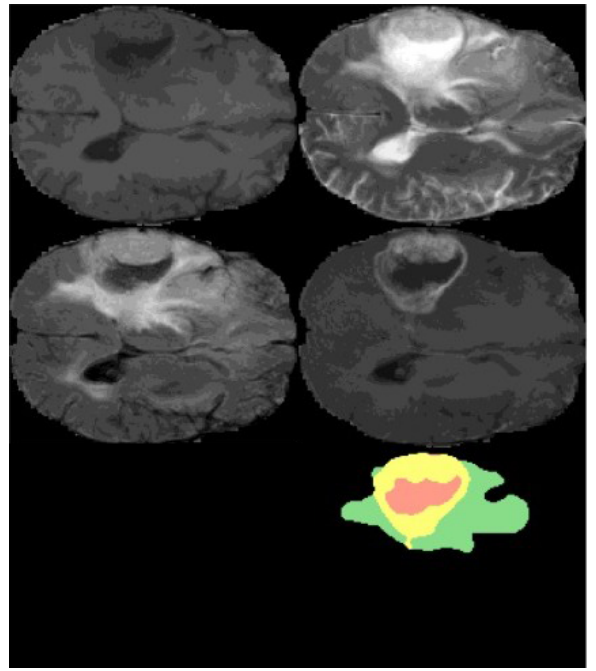

(a) Saggital View

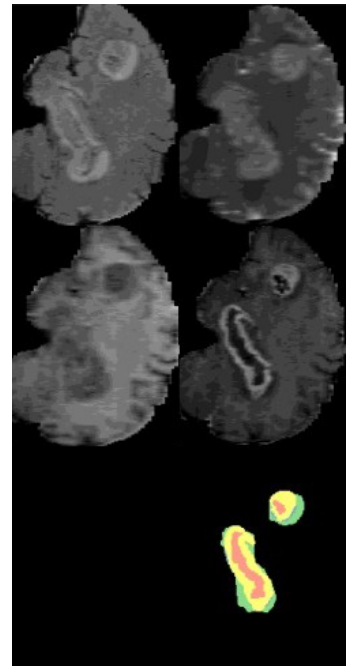

(b) Axial View

Fig. 2. Brain tumor segmentation based on deep neural network [25]

Unsupervised Learning. Unsupervised learning algorithms often target on building decision boundaries towards unlabeled training set. Clusters are then formed in the multidimensional feature vectors. K-means Clustering Methods [24] implement the hard segmentation for a certain number of K clusters while Fuzzy C-means [35] algorithms produce soft segmentations. It works by assigning the membership to pixels of the corresponding cluster in which they have maximum membership coefficients. Despite the advantages such as accuracy, efficiency and 
more image information presented, the final outcomes of unsupervised algorithms are constrained to the initial cluster matrix and feature extraction.

There is no unique standard segmentation algorithm could be applied to all the circumstances for all the organs, tissues or tumors. Some hybrid techniques could achieve better results. For instance, Unsupervised algorithm GA(Genetic Algorithm) derived from ANN are combined with Self-Organizing Maps (SOM) to detect the main features in medical images [27]. Table 2 compares the lists of current open-source tools for medical image segmentation.

Table 2. Segmentation Software Comparison

\begin{tabular}{|l|l|c|}
\hline Name & \multicolumn{1}{|c|}{ Characteristics } & Reference \\
\hline ITK-SNAP & $\begin{array}{l}\text { 1. Easy to learn and use software } \\
\text { 2. Rely on well-defined boundaries and homogeneous structure }\end{array}$ & {$[12]$} \\
\hline MITK & $\begin{array}{l}\text { 1. General and Extensible } \\
\text { 2. Less suitable for complicated anatomy structure }\end{array}$ & {$[45]$} \\
\hline 3D Slicer & $\begin{array}{l}\text { 1. Extensible } \\
\text { 2. Not time efficient } \\
\text { 3. Not appropriate for tissue with ill-boundaries and } \\
\text { inhomogeneous structure }\end{array}$ & {$[20]$} \\
\hline Seg 3D & $\begin{array}{l}\text { 1. Flexible } \\
\text { 2. Difficult to learn for non-professional user }\end{array}$ & {$[31]$} \\
\hline
\end{tabular}

\section{Mesh Generation}

In patient-specific modeling, computational mesh representation of anatomical structure is considered as the most crucial step, which contains discretization of multidimensional problems into fundamental geometry elements, such as tetrahedron or hexahedron [42]. Despite numerous automating algorithms have been developed, most of them target at engineering design and application, thus, are normally not suitable for anatomic modeling representation. Generating high quality three-dimensional computational finite element mesh from the segmented output for surgical simulation is facing many challenges. A good mesh should be accurately embodied for multi-material intersection mesh features: corners (none intersection), edges (1 intersection), and surfaces (2 intersections) [8]. As Vicecontl et al. [56] identified, four general rules should be followed for segmentation and mesh generation:

- Automation: Algorithms should be as automatic as possible to serve the patient-specific pipeline for any input patient data

- Geometrically Accuracy: Computational mesh simulation outcomes would be applied for the virtual surgery training, so that mesh generation must be geometric accurate 
- Robustness: Image information from CT or MRI and segmented output might be inadequate, complicated, or difficult to process. The mesh generation algorithm need to be robust enough to deal with these kinds of circumstances

- Generality: Quality mesh should be generated regardless of their geometric complexity

\subsection{Mesh Types}

Hexahedral Mesh. One hexahedral mesh needs to be represented by at least five tetrahedral meshes. The geometry structure of hexahedral mesh is more accurate, and volumetric locking free. However, it often requires a huge amount of time and operators even for a single mesh, consequently, by far, there are no automatic hexahedral mesh generation algorithms for the complicated human tissue modeling.

Meshless Methods. Finite element methods suffer from several technical limitations, for instance, huge computational cost of soft tissue deformation and accuracy of calculation relying on the generated mesh quality. Meshless methods are regarded as one possible solution to solve these technical difficulties. But in current stage, there is still a long gap before meshless algorithms are transferred to clinical practice.

Tetrahedral Mesh. Tetrahedral meshes are well accepted for patient-specific mesh generation because they are relatively easier to express complex geometry of organs and tissues like brain, heart, blood vessels, bones etc. If given the human organs or tissues geometric information in a surface manner, the tetrahedral meshes could be generated automatically. Unfortunately, tetrahedral elements present artificial stiffness known as volume locking when coping with incompressible materials, especially for brain [41] and soft tissues. Bonet and Burton proposed the average node pressure (ANP) tetrahedral mesh to achieve better results for nearly incompressible materials [9]. More recently, Leea et.al [34] present a robust and efficient form of the smoothed finite element method (SFEM) to prevent this issue.

\subsection{Tetrahedral Mesh Generation Strategy}

Tetrahedral elements are still the mainstream for the state of the art patient subject mesh expression. Mesh Generation strategies could be approximately divided into three groups: Advancing Front, Octree-based, and Delaunay Triangulations. Basic idea behind Advancing Front is to subdivide element by element iteratively to reduce the domain with an initial boundary mesh [54]. Mesh quality hugely relies on surface remeshing, and poor quality meshes are always produced along boundaries. Octree-based algorithms partition geometric cubes iteratively 
until touching the anticipated resolution [59] where multi-material junction consistency problem could be resolved. Tetrahedral meshes are produced from both the irregular cells, which is formed along with boundary and internal regular cells. However, orientation of the meshes changes with the octree cell. Quality improvements, namely local refinement, local remeshing, and Laplacian smoothing are often needed in the post-processing step.

Among three mesh generation strategies, only Delaunay based techniques offer the quality control and able to deal with arbitrary complexity anatomic tissue or organ structures Table 2 compares the properties of three mesh generation algorithms. Delaunay triangulation strategies could be further divided into three types:Boundary Constrained, Delaunay Refinement, and Voronoi-Delaunay:

Table 3. Three types of mesh generation algorithms applied in medical image

\begin{tabular}{|l|c|c|c|}
\hline & Surface Mesh Preserved & Quality Control & $\begin{array}{c}\text { Arbitrary Complexity } \\
\text { Input }\end{array}$ \\
\hline Advancing Front & $\checkmark$ & $\times$ & $\times$ \\
\hline Octree-based & $\times$ & $\times$ & $\times$ \\
\hline Delaunay Refinement & $\checkmark$ & $\checkmark$ & $\checkmark$ \\
\hline
\end{tabular}

Boundary Constrained The goal of boundary constrained is to keep the input boundary mesh unchanged [57]. In patient-specific solutions, this characteristic would be typically useful as it could maintain the intrinsic geometry attributes and save the operations like flips and vertex insertion. However, the complexity of human anatomy made the boundary-constrained algorithm hard to implement and can not guarantee the mesh quality.

Voronoi-Delaunay method creates a convex mesh-dependent energy function to ensure the local or global minimum exists [2]. High quality tetrahedral meshes with uniform distribution are produced and optimized at the same time, while the input boundary meshes must be changed, and implementation process is not computationally and timely efficient.

Delaunay Refinement aims at ensuring the mesh quality that leads the requirement of input boundary mesh modification. One way to achieve this goal is to keep the boundary elements while meshing the input domain after meshing or remeshing the input surface boundary. An alternative way is to operate the surface and volume mesh instantaneously by getting new boundary mesh through restricted Delaunay triangulation [53], which is a preferred solution for computational mesh generation.

There are several advantages of this technique. Firstly, instead of conventional mesh generation process who needs to go through a few tedious steps: 
isosurface extraction (usually done by marching cubes [37]), simplification, surface remeshing, merging and volume mesh generation, Delaunay refinement is an integrated process. Moreover, it gives the size and quality control for the tetrahedral mesh and approximation of surfaces and sharp features. Whereas two inevitable problems are also raised: small (dihedral) angles might destroy the refinement process, and ill-shaped tetrahedral (quasi-degenerate tetrahedral of special kind named Sliver) might be included in the final outcomes. Fig.3 is a 3D computational model generated from segmented image by CGAL [30]. From the review of current progress in mesh generation, it can be concluded that generating the high quality computational mesh and handling the boundary domain in a reasonable time frame is still very challenging.

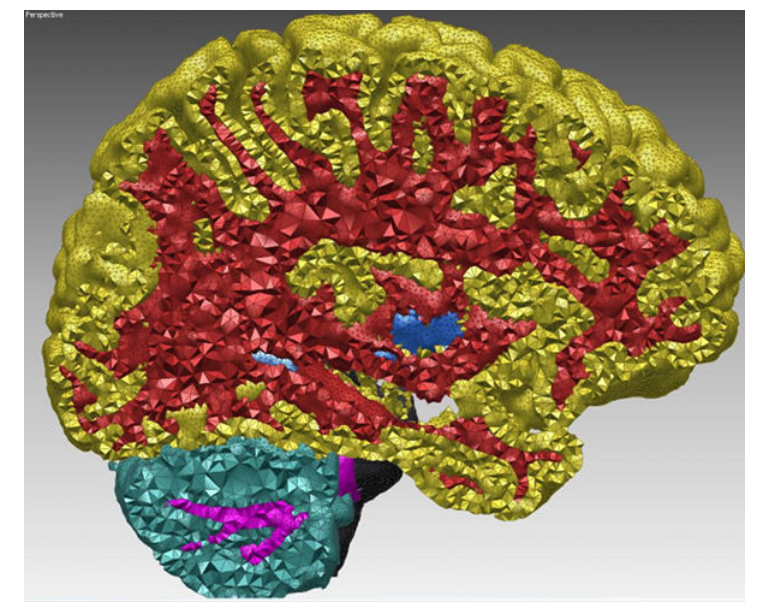

Fig. 3. Mesh Generation by Delaunay Refinement [30]

\section{Surgery Simulation}

Besides from precise anatomical modeling for a specific patient, real time soft tissue simulation is also a crucial part of the fast surgery simulation system [50]. Due to the fact that most of anatomical parts are soft tissue within the surgery, the accuracy of soft tissue deformation would enormously influence the performance of the whole framework [21]. There are mainly four types of simulation approaches have been widely used nowadays.

\subsection{Force Based Approach}

Mass-spring [5, 10,36], a simple and inexpensive scheme takes Newtons second law of motion as the theory basis to first compute the velocity from accelerations 
and then the positions from velocities, which is hard to tune the spring constants to get desired behavior and leads to overshooting problem.

\section{$5.2 \quad$ Structural Based Approaches}

Finite element method(FEM) [60] takes the continuum mechanics as the theory basis, which could produce accurate physical behaviors for different types of elastic material. However the model complexity makes the technique high computational cost, and the sparsity patterns from FEM are highly unstructured in $2 \mathrm{D}+$, thus it could be difficult to efficiently parallelize a FEM code [55].

\subsection{Position Based Dynamics}

Position Based Dynamics (PBD)[43] is a method works on positions directly in each simulation step. It is fast, stable, and controllable which makes the simulation process highly efficient, easier, and best suited for the interaction environment [7]. Even though the behavior of deformable objects can be modeled using PBD with additional constraints such as shape matching. It cannot accurately simulate the realistic behavior of soft tissues. The real-world human soft tissues produce non-linear and anisotropic behaviors or heterogeneous properties that hugely limits the results for surgery simulation. Although designing the complicated non-linearly constitutive model might solve these kinds of problems, this could also lead to high computational cost and difficult parameters. Data driven methods could be a possible solution.

\subsection{Data Driven Methods}

Geometric Data Driven Simulation [7] . captures the deformation example and decomposes the large scale geometry (the overall shape of the tissue) and the fine scale geometric (the details such as wrinkles). They can be represented as different resolutions, modeled separately, and connected by the subdivision schemes. Finally, high-resolution output with details is the synthesis of these two scales geometry, which could follow the position based dynamics method.

Mechanical Data Driven Simulation [47]. Unlike the geometric data driven methods where the parameter data could be directly measured from the training sets, mechanical data driven methods require both the deformation and forces data from the data captured stage, and parameter data are estimated through the numerical optimization based on physics mechanics (for example, FEM method)

\section{Future Challenges and Conclusion}

This paper takes a survey for current progress in virtual reality surgery simulation, particularly, concentrates on patient-specific modeling and real time soft 
tissue simulation. The complexity of human anatomical representation and the uniqueness for an individual patient and their ailments yield many challenges yet. Further works in these fields are desired, including accurately analyzing the geometric and anatomic structures from medical images, transferring the information to high quality computation models, and solving technical difficulties related to realistic soft tissue simulation and high fidelity natural human computer interactions, to present the trainee an immersive surgery environment with realistic visual and haptic feedback.

\section{Acknowledgements}

We would also like to thank the People Program (Marie Curie Actions) of the European Union's Seventh Framework Program FP7/2007-2013/ under REA grant agreement $n^{\circ}$ [612627] for their support.

\section{References}

1. Adams, R., Bischof, L.: Seeded region growing. IEEE Transactions on pattern analysis and machine intelligence 16(6), 641-647 (1994)

2. Alliez, P., Cohen-Steiner, D., Yvinec, M., Desbrun, M.: Variational tetrahedral meshing. In: ACM Transactions on Graphics (TOG). vol. 24, pp. 617-625. ACM (2005)

3. Antiga, L., Piccinelli, M., Botti, L., Ene-Iordache, B., Remuzzi, A., Steinman, D.A.: An image-based modeling framework for patient-specific computational hemodynamics. Medical \& biological engineering \& computing 46(11), 1097 (2008)

4. Badash, I., Burtt, K., Solorzano, C.A., Carey, J.N.: Innovations in surgery simulation: a review of past, current and future techniques. Annals of translational medicine 4(23) (2016)

5. Baraff, D., Witkin, A.: Large steps in cloth simulation. In: Proceedings of the 25th annual conference on Computer graphics and interactive techniques. pp. 43-54. ACM (1998)

6. Barratt, D.C., Chan, C.S., Edwards, P.J., Penney, G.P., Slomczykowski, M., Carter, T.J., Hawkes, D.J.: Instantiation and registration of statistical shape models of the femur and pelvis using $3 \mathrm{~d}$ ultrasound imaging. Medical image analysis 12(3), 358-374 (2008)

7. Bender, J., Koschier, D., Charrier, P., Weber, D.: Position-based simulation of continuous materials. Computers \& Graphics 44, 1-10 (2014)

8. Boltcheva, D., Yvinec, M., Boissonnat, J.D.: Mesh generation from 3d multimaterial images. Medical Image Computing and Computer-Assisted InterventionMICCAI 2009 pp. 283-290 (2009)

9. Bonet, J., Burton, A.: A simple average nodal pressure tetrahedral element for incompressible and nearly incompressible dynamic explicit applications. International Journal for Numerical Methods in Biomedical Engineering 14(5), 437-449 (1998)

10. Bouaziz, S., Martin, S., Liu, T., Kavan, L., Pauly, M.: Projective dynamics: fusing constraint projections for fast simulation. ACM Transactions on Graphics (TOG) $33(4), 154(2014)$ 
11. Bryson, S.: Virtual reality in scientific visualization. Communications of the ACM 39(5), 62-71 (1996)

12. Cevidanes, L.H., Tucker, S., Styner, M., Kim, H., Chapuis, J., Reyes, M., Proffit, W., Turvey, T., Jaskolka, M.: Three-dimensional surgical simulation. American journal of orthodontics and dentofacial orthopedics 138(3), 361-371 (2010)

13. Cootes, T.F., Edwards, G.J., Taylor, C.J.: Active appearance models. In: European conference on computer vision. pp. 484-498. Springer (1998)

14. Cootes, T.F., Taylor, C.J.: Active shape modelssmart snakes. In: BMVC92, pp. 266-275. Springer (1992)

15. Davis, J.E.: The use of simulation in causal analysis of sentinel events in healthcare. Ph.D. thesis, University of Pennsylvania (2016)

16. Duffy, A., Hogle, N., McCarthy, H., Lew, J., Egan, A., Christos, P., Fowler, D.: Construct validity for the lapsim laparoscopic surgical simulator. Surgical Endoscopy and Other Interventional Techniques 19(3), 401-405 (2005)

17. Endo, K., Sata, N., Ishiguro, Y., Miki, A., Sasanuma, H., Sakuma, Y., Shimizu, A., Hyodo, M., Lefor, A., Yasuda, Y.: A patient-specific surgical simulator using preoperative imaging data: an interactive simulator using a three-dimensional tactile mouse. Journal of Computational Surgery 1(1), 10 (2014)

18. Eschweiler, J., Stromps, J.P., Fischer, M., Schick, F., Rath, B., Pallua, N., Radermacher, K.: A biomechanical model of the wrist joint for patient-specific model guided surgical therapy: Part 2. Proceedings of the Institution of Mechanical Engineers, Part H: Journal of Engineering in Medicine 230(4), 326-334 (2016)

19. Eschweiler, J., Stromps, J.P., Fischer, M., Schick, F., Rath, B., Pallua, N., Radermacher, K.: Development of a biomechanical model of the wrist joint for patientspecific model guided surgical therapy planning: Part 1. Proceedings of the Institution of Mechanical Engineers, Part H: Journal of Engineering in Medicine 230(4), 310-325 (2016)

20. Fedorov, A., Beichel, R., Kalpathy-Cramer, J., Finet, J., Fillion-Robin, J.C., Pujol, S., Bauer, C., Jennings, D., Fennessy, F., Sonka, M., et al.: 3d slicer as an image computing platform for the quantitative imaging network. Magnetic resonance imaging 30(9), 1323-1341 (2012)

21. Gallagher, A.G., Ritter, E.M., Champion, H., Higgins, G., Fried, M.P., Moses, G., Smith, C.D., Satava, R.M.: Virtual reality simulation for the operating room: proficiency-based training as a paradigm shift in surgical skills training. Annals of surgery 241(2), 364-372 (2005)

22. Goel, V.R., Greenberg, R.K., Greenberg, D.P.: Mathematical analysis of dicom ct datasets: can endograft sizing be automated for complex anatomy? Journal of vascular surgery 47(6), 1306-1312 (2008)

23. Gonzalez, R., Wintz, P.: Digital image processing (1977)

24. Hartigan, J.A., Wong, M.A.: Algorithm as 136: A k-means clustering algorithm. Journal of the Royal Statistical Society. Series C (Applied Statistics) 28(1), 100108 (1979)

25. Havaei, M., Davy, A., Warde-Farley, D., Biard, A., Courville, A., Bengio, Y., Pal, C., Jodoin, P.M., Larochelle, H.: Brain tumor segmentation with deep neural networks. Medical image analysis 35, 18-31 (2017)

26. Heimann, T., Meinzer, H.P.: Statistical shape models for $3 \mathrm{~d}$ medical image segmentation: a review. Medical image analysis 13(4), 543-563 (2009)

27. Indira, S., Ramesh, A.: Image segmentation using artificial neural network and genetic algorithm: a comparative analysis. In: Process Automation, Control and Computing (PACC), 2011 International Conference on. pp. 1-6. IEEE (2011) 
28. Iwamoto, N., Shum, H.P., Yang, L., Morishima, S.: Multi-layer lattice model for real-time dynamic character deformation. In: Computer Graphics Forum. vol. 34, pp. 99-109. Wiley Online Library (2015)

29. Jain, R., Kasturi, R., Schunck, B.G.: Machine vision, vol. 5. McGraw-Hill New York (1995)

30. Jamin, C., Alliez, P., Yvinec, M., Boissonnat, J.D.: Cgalmesh: a generic framework for delaunay mesh generation. ACM Transactions on Mathematical Software (TOMS) 41(4), 23 (2015)

31. Johnson, C.: Biomedical visual computing: Case studies and challenges. Computing in science \& engineering 14(1), 12-21 (2012)

32. Kent, D.M., Hayward, R.A.: Limitations of applying summary results of clinical trials to individual patients: the need for risk stratification. Jama 298(10), 1209$1212(2007)$

33. Lai, J.Y., Essomba, T., Lee, P.Y., et al.: Algorithm for segmentation and reduction of fractured bones in computer-aided preoperative surgery. In: Proceedings of the 3rd International Conference on Biomedical and Bioinformatics Engineering. pp. 12-18. ACM (2016)

34. Leea, C.K., Mihaib, L.A., Halec, J.S., Kerfridena, P., Bordasc, S.P.: Strain smoothing for compressible and nearly-incompressible finite elasticity. Computers \& Structures (2016)

35. Lei, T., Sewchand, W.: Statistical approach to x-ray ct imaging and its applications in image analysis. ii. a new stochastic model-based image segmentation technique for x-ray ct image. IEEE Transactions on Medical Imaging 11(1), $62-69$ (1992)

36. Liu, T., Bargteil, A.W., O'Brien, J.F., Kavan, L.: Fast simulation of mass-spring systems. ACM Transactions on Graphics (TOG) 32(6), 214 (2013)

37. Lorensen, W.E., Cline, H.E.: Marching cubes: A high resolution 3d surface construction algorithm. In: ACM siggraph computer graphics. vol. 21, pp. 163-169. ACM (1987)

38. Makiyama, K., Nagasaka, M., Inuiya, T., Takanami, K., Ogata, M., Kubota, Y.: Development of a patient-specific simulator for laparoscopic renal surgery. International Journal of Urology 19(9), 829-835 (2012)

39. Marr, D., Hildreth, E.: Theory of edge detection. Proceedings of the Royal Society of London B: Biological Sciences 207(1167), 187-217 (1980)

40. Mihalef, V., Ionasec, R.I., Sharma, P., Georgescu, B., Voigt, I., Suehling, M., Comaniciu, D.: Patient-specific modelling of whole heart anatomy, dynamics and haemodynamics from four-dimensional cardiac ct images. Interface Focus 1(3), 286-296 (2011)

41. Miller, K.: Biomechanics of brain for computer integrated surgery. Warsaw University of Technology Publishing House Warsaw, Poland (2002)

42. Mohamed, A., Davatzikos, C.: Finite element mesh generation and remeshing from segmented medical images. In: Biomedical Imaging: Nano to Macro, 2004. IEEE International Symposium on. pp. 420-423. IEEE (2004)

43. Müller, M., Heidelberger, B., Hennix, M., Ratcliff, J.: Position based dynamics. Journal of Visual Communication and Image Representation 18(2), 109-118 (2007)

44. Neal, M.L., Kerckhoffs, R.: Current progress in patient-specific modeling. Briefings in bioinformatics 11(1), 111-126 (2010)

45. Nolden, M., Zelzer, S., Seitel, A., Wald, D., Müller, M., Franz, A.M., Maleike, D., Fangerau, M., Baumhauer, M., Maier-Hein, L., et al.: The medical imaging interaction toolkit: challenges and advances. International journal of computer assisted radiology and surgery 8(4), 607-620 (2013) 
46. de Oliveira, J.E., Giessler, P., Deserno, T.M.: Patient-specific anatomical modelling. In: E-Health and Bioengineering Conference (EHB), 2015. pp. 1-4. IEEE (2015)

47. Otaduy, M.A., Bickel, B., Bradley, D., Wang, H.: Data-driven simulation methods in computer graphics: cloth, tissue and faces. In: ACM SIGGRAPH 2012 Courses. p. 12. ACM (2012)

48. OReilly, M.A., Whyne, C.M.: Comparison of computed tomography based parametric and patient-specific finite element models of the healthy and metastatic spine using a mesh-morphing algorithm. Spine 33(17), 1876-1881 (2008)

49. Pal, N.R., Pal, S.K.: A review on image segmentation techniques. Pattern recognition 26(9), 1277-1294 (1993)

50. Pan, J.J., Chang, J., Yang, X., Liang, H., Zhang, J.J., Qureshi, T., Howell, R., Hickish, T.: Virtual reality training and assessment in laparoscopic rectum surgery. The International Journal of Medical Robotics and Computer Assisted Surgery 11(2), 194-209 (2015)

51. Prewitt, J.M.: Object enhancement and extraction. Picture processing and Psychopictorics 10(1), 15-19 (1970)

52. Ricotta, J.J., Pagan, J., Xenos, M., Alemu, Y., Einav, S., Bluestein, D.: Cardiovascular disease management: the need for better diagnostics. Medical \& biological engineering \& computing 46(11), 1059-1068 (2008)

53. Rineau, L., Yvinec, M.: Meshing 3 d domains bounded by piecewise smooth surfaces. In: Proceedings of the 16th International Meshing Roundtable. pp. 443-460. Springer (2008)

54. Schöberl, J.: Netgen an advancing front $2 \mathrm{~d} / 3 \mathrm{~d}$-mesh generator based on abstract rules. Computing and visualization in science 1(1), 41-52 (1997)

55. Sifakis, E., Barbic, J.: Fem simulation of 3d deformable solids: a practitioner's guide to theory, discretization and model reduction. In: ACM SIGGRAPH 2012 Courses. p. 20. ACM (2012)

56. Viceconti, M., Davinelli, M., Taddei, F., Cappello, A.: Automatic generation of accurate subject-specific bone finite element models to be used in clinical studies. Journal of biomechanics 37(10), 1597-1605 (2004)

57. Weatherill, N.P., Hassan, O.: Efficient three-dimensional delaunay triangulation with automatic point creation and imposed boundary constraints. International Journal for Numerical Methods in Engineering 37(12), 2005-2039 (1994)

58. Zhang, A., Hünerbein, M., Dai, Y., Schlag, P.M., Beller, S.: Construct validity testing of a laparoscopic surgery simulator (lap mentor R). Surgical endoscopy 22(6), 1440-1444 (2008)

59. Zhang, Y., Hughes, T.J., Bajaj, C.L.: An automatic 3d mesh generation method for domains with multiple materials. Computer methods in applied mechanics and engineering 199(5), 405-415 (2010)

60. Zienkiewicz, O.C., Taylor, R.L.: The finite element method for solid and structural mechanics. Butterworth-heinemann (2005) 